\title{
Modeling Prioritized Broadcasting in Multichannel Vehicular Networks
}

\author{
Claudia Campolo, Student Member, IEEE, Antonella Molinaro, Member, IEEE, \\ Alexey Vinel, Member, IEEE, and Yan Zhang, Senior Member, IEEE
}

\begin{abstract}
Effective data broadcasting is essential in vehicular networks not only for road-safety message dissemination but also to aid routing and cooperative driving applications through periodic beaconing and to spread network initialization advertisements that are mandatory to support infotainment applications. Broadcast data are neither acknowledged nor retransmitted in case of failure, which raises the possibility of frame loss due to channel errors and collisions with multiple simultaneous broadcasts. This paper aims at modeling periodic broadcasting on the control channel of IEEE Std. 802.11p vehicular networks with multichannel architecture. Unlike previous related work, the proposed novel analytical approach accounts for mutual influence among nodes, frequent periodic updates of broadcasted data, standard network advertisement procedures, and 802.11p prioritized channel access with multichannel-related phenomena under various link quality conditions.
\end{abstract}

Index Terms-Broadcast, vehicular networks, wireless access in vehicular environments (WAVE), 802.11p.

\section{INTRODUCTION}

$\mathbf{V}$ EHICULAR ad hoc networks (VANETs) can greatly benefit from recent advances in transportation telematics and wireless technologies that make vehicle-to-vehicle $(\mathrm{V} 2 \mathrm{~V})$ and vehicle-to-infrastructure communications feasible while enabling a plethora of new intelligent transportation system services for drivers and the vehicle's passengers. Applications span from emergency services, like road-safety and driverassistance applications, automatic crash notification, or hazardous road condition reports, to various convenience and commercial applications, like mobile office and information advertisements, envisioned for future automobiles [1]. Traffic monitoring and management can also be supported by vehicular communications (e.g., adaptive cooperative cruise control) to improve traffic flow efficiency and reduce congestion.

IEEE Std. 802.11p [2] has recently been ratified as a standard to provide wireless access in vehicular environments (WAVE). It is based on the prioritized enhanced distributed channel

Manuscript received February 13, 2011; revised October 5, 2011; accepted November 27, 2011. Date of publication December 23, 2011; date of current version February 21, 2012. The review of this paper was coordinated by Dr. P. Lin.

C. Campolo and A. Molinaro are with the Dipartimento di Informatica, Matematica, Elettronica e Trasporti, University Mediterranea of Reggio Calabria, 89100 Reggio Calabria, Italy (e-mail: claudia.campolo@unirc.it; antonella.molinaro@unirc.it).

A. Vinel is with the Department of Communications Engineering, Tampere University of Technology, 33720 Tampere, Finland (e-mail: vinel@ ieee.org).

Y. Zhang is with the Simula Research Laboratory, 1364 Fornebu, Norway (e-mail: yanzhang@ieee.org).

Color versions of one or more of the figures in this paper are available online at http://ieeexplore.ieee.org.

Digital Object Identifier 10.1109/TVT.2011.2181440 access (EDCA) and the multichannel architecture specified in IEEE 1609.4 [3], which uses a common control channel $(\mathrm{CCH})$ for signaling and safety-critical data exchange and up to six service channels (SCHs) for nonsafety (e.g., comfort and infotainment) data exchange. Periodical and synchronous switching between $\mathrm{CCH}$ and $\mathrm{SCH}$ is mandatory for single-radio devices.

Most of the applications envisioned for vehicular networks rely on one-hop data broadcasting over $\mathrm{CCH}$. Applications for driver assistance and cruise control require periodic broadcasting of beacons to discover nearby vehicles and to advertise the vehicle's position and velocity. Beacons are also essential to support smart multihop dissemination strategies for safety warning messages. Drive-thru Internet access and infotainment services rely on preliminary WAVE service advertisement (WSA) broadcasting to initialize the network that provides the announced services. Beacons and WSAs are periodically and massively transmitted by vehicles under normal operational conditions and constitute the main cause of traffic load on $\mathrm{CCH}$.

Broadcast frames cannot be acknowledged so failed transmissions cannot be detected by the sender and backoff adaptation mechanisms cannot be used, thus leading to poor performances due to collisions, particularly if several neighboring nodes are contending to seize the channel [4]. Because of channel switching, a spike in frame collisions very likely occurs at the beginning of the $\mathrm{CCH}$ interval. Moreover, due to time relevance of the carried information, WSAs and beacons are normally dropped at the end of the channel interval due to lifetime expiration.

The main focus of this paper is the design of an innovative analytical model for understanding periodic broadcasting of regular traffic (i.e., beacons and WSAs) on CCH in VANETs. It goes beyond previous related work since it simultaneously accounts for some of the following unique features of 802.11p/WAVE networks that are expected to strongly affect performance:

1) alternating channel switching of single-radio devices due to the WAVE multichannel architecture and related phenomena, like synchronized frame collisions at the $\mathrm{CCH}$ interval beginning and frame dropping due to time expiring at the $\mathrm{CCH}$ interval end;

2) critical role of traffic routinely broadcasted on $\mathrm{CCH}$ under normal network operation, like beacons and WSAs, crucial for the support of cooperative driving and routing and for network initialization advertisements, respectively;

3) differentiated access priorities for beacons and WSAs that closely follow suggestions on EDCA access categories (ACs) given in IEEE standard documents [2], [3]; 
4) short time relevance of the information carried by beacons and WSAs, which ask for dropping unsent frames at the end of a $\mathrm{CCH}$ interval, and periodic information update, which would benefit from freshly generated frames at the beginning of every $\mathrm{CCH}$ interval.

As a consequence of the last item, the proposed model focuses on non-saturation conditions and uses frame delivery probability as the main performance metric instead of traditional queuing delay that is of less interest due to the short-lived nature of frames in the scenario under investigation.

Unlike traditional models for 802.11 network analysis, our analytical approach disregards the common assumption about the independent operation of an arbitrary node, which demonstrated its validity for saturation cases, under high number of nodes and large contention windows $C W$ [5]. The model presented here does not require any approximate derivations and precisely describes the underlying multidimensional stochastic process, which leads to a perfect match of the proposed analytical approach with simulations for a wide spectrum of input parameter values. In our preliminary work [6], we modeled broadcast transmissions over $\mathrm{CCH}$ by accounting for the channel switching effect under variable traffic load and ideal channel conditions but without accounting for EDCA differentiation among traffic classes. In this paper, we intend to take a step forward and to model EDCA prioritization of beacons and WSAs by clearly identifying causes of frame losses under various error-prone radio link conditions and transmission data rates.

The contributions of this paper can be summarized as follows.

1) We present a novel analytical model for broadcasting in IEEE $802.11 \mathrm{p} /$ WAVE that overcomes the shortcomings of existing ones by jointly covering the features of multichannel protocol operation with the prioritizations of messages broadcasted on the $\mathrm{CCH}$.

2) We conduct performance evaluation of beacon and WSA broadcasting on the $\mathrm{CCH}$ interval under wide diversity of protocol and network parameter values, with a focus on understanding the effects of prioritization.

3) We quantitatively characterize the negative influences of channel switching on the vehicular network performance and bring some insight into ways to combat them.

This paper is organized as follows: Section II gives an overview of the standards IEEE 802.11p and IEEE 1609.4 and details about the broadcast traffic that is the focus of our study. Section III discusses motivations for the research and summarizes the related work on analytical models for $802.11 \mathrm{p}$. The proposed analytical framework is described in Section IV; its validation and related performance analysis is reported in Section V. Final remarks are summarized in Section VI.

\section{BROADCASTING IN IEEE 802.11p/WAVE}

\section{A. IEEE 802.11p/WAVE and the Multichannel Architecture}

The IEEE 802.11p [2] has been ratified in July 2010 to support WAVE. The prioritized EDCA scheme is the core of the medium access control (MAC) layer. It provides differentiated access by assigning to traffic belonging to each of the four ACs a set of distinct channel access parameters, including arbitration interframe space (AIFS) and $C W$ range (that is, minimum $C W \min$ and maximum $C W \max$ values). The four classes are referred to as background (AC_BK), best effort (AC_BE), video ( $\left.\mathrm{AC}_{-} \mathrm{VI}\right)$, and voice $\left(\mathrm{AC}_{-} \mathrm{VO}\right)$ with increasing priority. Higher priority ACs have smaller $C W \mathrm{~s}$ and shorter AIFSs.

The EDCA scheme relies on the carrier sense multiple access with collision avoidance (CSMA/CA) technique according to which a station that desires to transmit must probe the medium before transmission to determine whether it is busy or not. In particular, a wireless station, with a backlogged flow of class AC, will sense the medium idle for $A I F S[A C]$ before attempting to transmit a frame: $A I F S[A C]=a S I F$ STime + $A I F S N[A C] \times$ aSlotime, where $A I F S N[A C]$ is an integer number, aSlotTime is the duration of a slot time interval, and aSIF STime is the short interframe space (SIFS) duration, i.e., the shortest time interval between two consecutive frames on the channel. If the medium is detected as busy, then the station will defer its transmission of an additional backoff time to avoid collisions between multiple nodes that have been deferring to the same event. The backoff is computed as a random number of slot times selected from a uniform distribution over the interval $[0, C W[A C]]$. At the first transmission attempt, $C W[A C]$ takes $C W \min [A C]$ as an initial value, and it is doubled at every retransmission with an upper limit equal to $C W \max [A C]$. The backoff counter is decremented at the end of each slot while the medium is sensed idle; if, during the backoff countdown, the channel is sensed as busy at any slot interval, then the countdown is frozen, and it will be resumed when the channel is determined to be idle for an $A I F S[A C]$ period. The frame is transmitted when the backoff timer reaches zero.

All successfully received unicast data frames must be acknowledged by the receiver. It transmits an acknowledgement (ACK) frame SIFS seconds after the data frame has been received. Retransmission is scheduled by the sender if no ACK is received. When a frame is not correctly received, due to collision or channel errors, the receiver will not send back the ACK and will sense the medium idle for an extended interframe space (EIFS), ${ }^{1}$ instead of an $A I F S$, before transmitting on its turn.

Unlike unicast traffic, broadcast frames are never acknowledged by the receivers; therefore, failed transmissions cannot be detected by the sender, and broadcast frames cannot be retransmitted. As a consequence, the backoff always takes values in the range $[0, C W \min [A C]]$.

IEEE 1609.4 [3] specifies the multichannel operation on top of the 802.11p MAC layer. The channel time is divided into synchronization intervals with a fixed length of $100 \mathrm{~ms}$, consisting of a $\mathrm{CCH}$ interval and a $\mathrm{SCH}$ interval, as shown in Fig. 1. A global time reference, the coordinated universal time provided by navigation satellite systems, is used for time synchronization.

\footnotetext{
${ }^{1} \operatorname{EIFS}[A C]$ is equal to (aSIFSTime $\left.+A I F S[A C]+A C K T x T i m e\right)$, where ACKT TTime is the ACK frame transmission time.
} 


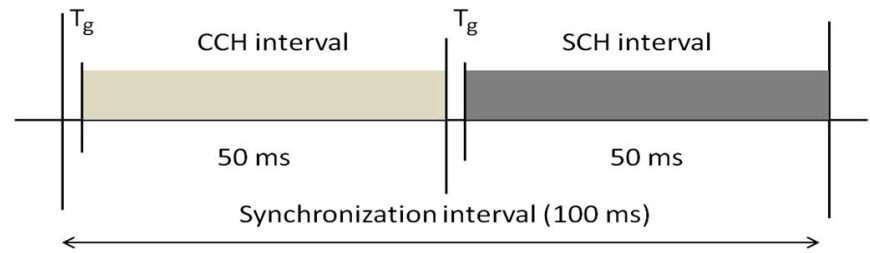

Fig. 1. WAVE 1609.4 multichannel operation.

Single-radio WAVE devices, which are expected to spread in the first deployment phase of VANETs, alternatively tune into $\mathrm{CCH}$ and $\mathrm{SCH}$ during the synchronization interval; during the $\mathrm{CCH}$ interval, the channel activity on all SCHs is suspended and vice versa.

To prevent multiple devices from transmitting simultaneously upon switching, a 4-ms guard time ( $T_{g}$ in Fig. 1) that accounts for radio switching delay and timing inaccuracies is left free at the beginning of each interval. During $T_{g}$, no transmission is allowed; the medium is declared busy so that all backlogged stations will undergo random backoff before attempting to access the channel. However, by doing so, synchronized frame collisions at the channel interval (both $\mathrm{CCH}$ and $\mathrm{SCH}$ ) beginning could still occur among nodes extracting the same backoffs [7]. Collisions increase with the number of devices, the frame inter-arrival rate, and the volume of data. The phenomenon is exacerbated if an application is unaware of channel switching, i.e., it continues generating data also during the "wrong" interval when it cannot be transmitted, and the generated data will be buffered at the MAC layer waiting for the "right" channel interval before being transmitted.

The occurrence of synchronized collisions could be reduced if the application layer becomes aware of the channel switching and passes data to the MAC layer only during the proper channel interval.

In addition to synchronized frame collisions, mandatory channel switching is also the cause of another type of event that is unique to WAVE networks: a frame cannot be transmitted if the residual time before the end of a channel interval is shorter than its transmission delay. Such a frame can be queued waiting for the next channel interval or dropped in case its lifetime is exceeded. Both types of frame loss events, due to synchronized frame collisions at the channel interval beginning and induced by channel expiry time at the channel interval end, are particularly detrimental to time-critical broadcast traffic. They will be explicitly considered in the model proposed in this paper.

\section{B. Data Broadcasting on $\mathrm{CCH}$}

$\mathrm{CCH}$ is destined to transport both safety-critical messages and regular traffic like WSAs and beacons. Safety message transmission is triggered by the occurrence of hazardous events on the road, whereas regular messages are periodically and routinely transmitted on the $\mathrm{CCH}$. They represent the traffic load over $\mathrm{CCH}$ under normal operational conditions.

Beacons are short status messages used by all vehicles to announce themselves to one-hop neighbors; they carry status information about the vehicle type, position, speed, and direction. Beacons are mandatory to support cooperative vehicular applications, such as collision avoidance, driver assistance, and cruise control, which require accurate and timely context awareness. They are also crucial to offer a neighbor discovery service to proactive routing algorithms or to smart safety dissemination strategies [8], [9].

To meet these applications' requirements, the beacon generation rate is typically $10 \mathrm{~Hz}$ [10], that is, once every WAVE synchronization interval. This implies high load over the channel when the number of vehicles is high. In the literature, we find examples of adaptation of the beacon generation rate and transmission power [8], [10], [11] to reduce the channel load while ensuring accurate and timely neighborhood awareness. Nevertheless, in the remainder of this paper, we consider a fixed $10-\mathrm{Hz}$ beacon generation rate to study worst-case system performances.

Even if beacons are not explicitly mentioned in $802.11 \mathrm{p} /$ WAVE standards, they are cited as heartbeat messages by Dedicated Short-Range Communications (DSRC) in [12] and as cooperative awareness messages by the European Telecommunications Standards Institute (ETSI) in [13]. On the other hand, WSAs are explicitly mentioned by 1609.4 as a means to advertise initialization of a basic service set (BSS). WSAs contain information on offered service and network parameters necessary to join the advertised BSS (BSS identifier, EDCA parameters set, selected $\mathrm{SCH}$, etc.). WAVE providers, i.e., either road-side units (RSUs) or vehicles [on board units (OBUs)], announce their BSS set up by periodically broadcasting WSAs to one-hop far vehicles during the $\mathrm{CCH}$ interval. WAVE users interested in the announced services simply switch on the advertised SCH during the next channel interval and join the BSS. A failure in the WSA reception prevents successive nonsafety data exchange on $\mathrm{SCH}$, given that potential users are unaware of the BSS setup.

\section{Motivations AND Related Work}

\section{A. Motivations}

Vehicular networks have some of the following peculiarities that make previous analytical models of generic 802.11 networks unsuitably matched.

1) Multichannel architecture. $802.11 \mathrm{p}$ is designed to work on multiple channels, and therefore, analytical models should take into account channel switching and the related phenomena of synchronized frame collisions and losses induced by channel expiry time.

2) Differentiated channel access. The heterogeneous requirements of vehicular applications make prioritized access mandatory in vehicular networks. More aggressively differentiated priorities are set in 802.11p/WAVE [2], [3] compared with generic 802.11 networks.

3) Central role of broadcasting. Unlike 802.11 modeling that typically considers unicast data exchange, there is rationale for analyzing broadcasting in $802.11 \mathrm{p}$ networks, given its role of a basic and crucial communication mode for several road-safety and traffic-efficiency applications.

4) Nonsaturation conditions. While most of the traditional models focus on saturated conditions, for typical arrival 
rates and delay requirements of broadcast data on $\mathrm{CCH}$, a vehicular network operates far from saturation with no queues in the MAC layer.

5) Periodic broadcasting. Beacons and WSAs are periodically broadcasted (typically once per $\mathrm{CCH}$ interval), which makes commonly used Poisson arrival process assumption not feasible.

6) Performance metrics. Mean packet queuing delay is the traditional performance metric in the nonsaturated case; but given the time-critical broadcast traffic under analysis, the focus must be on successful frame delivery probability as the main performance metric.

Some of these issues have separately been considered in the related literature, but they have not all been simultaneously modeled. The novelty of our approach comes from the fact that the proposed analytical model contemporaneously covers all such features.

An important fact is that our analytical method does not adopt the widely used simplifying (and quite limiting [5]) assumption about the independent operation of an arbitrary node and constant channel access probability, but precisely models underlying stochastic process covering mutual influence among nodes. This fact allows our model to be exact for a wide spectrum of input parameters, including a small number of nodes, small contention windows, etc. Moreover, we believe that the presented approach has a great potential to be extended for more complex vehicular scenarios.

\section{B. Related Work}

In [14], a 1-D discrete-time Markov chain is proposed for packet delivery and delay performance evaluation of emergency messages and beacons under saturated and unsaturated conditions. Traffic prioritization is only based on contention window differentiation while ignoring AIFS, as instead done in [15], where a 2-D embedded Markov chain models the impact of differentiated AIFSs. However, compared with [14], the work models ideal wireless channel where packet loss can be only due to collisions, and assumes load saturation conditions.

In [16], a Markov chain is used for modeling V2V communications under both saturated and unsaturated conditions. It investigates the influence of the beacon generation rate on the successful data delivery and transmission delay. In [17], a simplified model of the 802.11 distributed channel function (DCF) is used to analyze beaconing in vehicular networks and calculate the probability of collision-free transmissions. A transmission can fail because of direct collisions or interference from hidden nodes. In [18], an analytical model of DCF is proposed for the evaluation of safety data performances in an unsaturated network of vehicles on a highway. Vehicles are modeled as nodes in a 1-D network, which are stationary during the communication interval. Packet generation follows a Poisson distribution so that each vehicle can be modeled as a M/G/1 queue, where no packet loss can occur because of buffer overflow. The work in [19] provides an analytical study on broadcasting performance on $\mathrm{CCH}$ in $802.11 \mathrm{p}$ networks, which captures the EDCA traffic prioritization. To account for the fact that broadcast frames are not retransmitted, the proposed discrete-time Markov chains only have one backoff stage.

Although all the aforementioned works can be considered as pioneering in the field of $802.11 \mathrm{p}$ analytical modeling, they do not take into account WAVE channel switching, which is expected to strongly penalize network performance, particularly for broadcast traffic. There are just a few examples of works that have analytically investigated such an issue [6], [20], [21]. In [20], an approximate analytical model is proposed to evaluate throughput and collision probability in an 802.11 petwork, where nodes always have packets to send. The model roughly estimates the saturation throughput without considering packet differentiation. In [21], the performance of the WAVE channel coordination mechanism under different traffic patterns and channel interval durations is analytically investigated by combining probabilistic analysis, M/G/1 queuing, and EDCA Markov chain. Unsaturated conditions are modeled, according to which a single priority class at each node generates Poisson-distributed packets. Attention is focused on unicast transmissions; on the contrary, broadcasting is the focus in our paper. In [6], we presented a preliminary analytical model for broadcasting over $\mathrm{CCH}$ of $802.11 \mathrm{p}$ networks that explicitly takes into account the WAVE channel switching. Compared with our previous work, two main additional features are considered here: 1) broadcast traffic prioritization and 2) error-prone channel conditions.

\section{Analytical Framework}

\section{A. Model Assumptions}

Vehicular nodes are assumed to be equipped with singleradio OBUs, which access the channel according to the alternating scheme: every $50 \mathrm{~ms}$, they switch between $\mathrm{CCH}$ and $\mathrm{SCH}[3]$.

The focus of the model is on analyzing the broadcasting performance on $\mathrm{CCH}$ of a VANET under normal working conditions, when only periodic broadcast messages, like beacons and WSAs, are frequently and massively transmitted. Evaluation of their performance is of the highest practical importance in a VANET. Event-based safety-critical messages transmitted only in case of emergency are out of the scope of this study.

The considered application layer, in charge of generating WSAs and beacons, is aware of the channel switching; this implies that messages are sent to the MAC layer only during the $\mathrm{CCH}$ interval. A worst-case scenario is considered for message generation: Every node has one frame: either a beacon (in case of a WAVE user) or a WSA (in case of a WAVE provider $^{2}$ ), ready to be transmitted at the beginning of every $\mathrm{CCH}$ interval. ${ }^{3}$ This assumption is consistent with the ETSI requirements in [13] that suggest beacons to be broadcasted with a $10-\mathrm{Hz}$ frequency to provide timely and accurate neighborhood awareness. It is also consistent with the indications in [3] that foresee

\footnotetext{
${ }^{2} \mathrm{~A}$ WAVE provider does not need sending beacons, since WSAs include its position and kinematics data.

${ }^{3} \mathrm{Of}$ course, the actual instant of transmission in the $\mathrm{CCH}$ interval is randomly determined by the extracted backoff according to EDCA rules.
} 
the BSS initialization parameters in WSAs to be changed on a synchronization interval basis.

Due to the bounded lifetime of beacons (100 ms [13]) and WSAs (one $\mathrm{CCH}$ interval), it is reasonable to assume that each nontransmitted frame is dropped from the MAC layer buffer at the end of every $\mathrm{CCH}$ interval.

A simple noise model is considered, which is commonly used for MAC analysis in error-prone channels. Bit errors are independent and occur with a fixed bit error rate (BER); the header is always received successfully, while even a 1-bit error in the payload destroys the whole frame.

The following common simplifying assumptions also hold in the analytical framework.

1) All nodes are within reciprocal communication range (no hidden terminals are modeled).

2) The carrier sense range of each node coincides with the communication range.

3) The mobility of nodes is not considered, since we can reasonably assume that the one-hop neighborhood of a given node does not significantly change in the short time span of our interest (a CCH interval). ${ }^{4}$ More precisely, the requirement of the model is only that the neighborhood does not change during a frame transmission time.

This makes the assumption of ignoring mobility even more reasonable.

\section{B. Model Description}

For the reader's convenience, the main notations of the analytical model are summarized in Table I.

Events occur on the $\mathrm{CCH}$ interval on a discrete slotted time scale, with slot time duration equal to $\sigma$. The radio channel can be in one of three possible states: idle, success, or failure; they, respectively, model the cases of lack of transmission, only one transmission, and multiple simultaneous transmissions (i.e., collision) or one corrupted transmission.

Without loss of generality, two types of nodes are modeled that respectively transmit types 1 and 2 frames over $\mathrm{CCH}$ during normal working conditions. These frames represent beacons and WSAs. Notwithstanding, the proposed approach can easily be generalized to operate with more than two types of nodes and more than two access priorities.

The main parameters distinguishing the two frame types are the following:

1) Type 1: $N_{1}$ (number of nodes), $L_{1}$ (frame length), $W_{1}$ (minimum CW size), $\mathrm{AIFS}_{1}$

2) Type 2: $N_{2}$ (number of nodes), $L_{2}$ (frame length), $W_{2}$ (minimum CW size), $\mathrm{AIFS}_{2}$.

We assume that $\mathrm{AIFS}_{1} \leq \mathrm{AIFS}_{2}$ and set $\Delta=\left(\mathrm{AIFS}_{2}-\right.$ $\left.\mathrm{AIFS}_{1}\right) / \sigma$ as the number of slots after a busy medium when

\footnotetext{
${ }^{4}$ In $50 \mathrm{~ms}$, vehicles move a very few meters forward (typically, less than $1 \mathrm{~m}$ in a city and less than $2 \mathrm{~m}$ on a highway). Even considering vehicles moving in opposite directions, the travelled space in $50 \mathrm{~ms}$ is still short; for example, on a highway, vehicles travelling at $130 \mathrm{~km} / \mathrm{h}$ move $3.6 \mathrm{~m}$ apart. Vehicles often travel at similar speed, in a platoon-like pattern, so that considering the one-hop neighborhood of a vehicle not changing during a $\mathrm{CCH}$ interval is reasonable.
}

TABLE I

Main Notations for the AnALytical Model

\begin{tabular}{cc}
\hline \hline Notation & Definition \\
\hline$A I F S_{y}$ & AIFS for type $y$ frames \\
$B E R$ & probability of a bit to be in error \\
$c_{y}$ & slotted collision time duration for type $y$ frames as \\
& observed by nodes of type 1
\end{tabular}

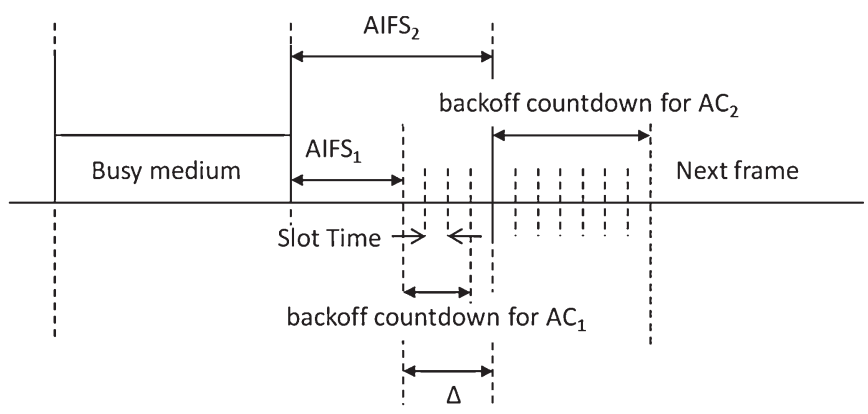

Fig. 2. AIFS and backoff differentiation.

type 1 nodes have exclusive right to access the channel, as illustrated in Fig. 2. The same $\Delta$ value is used both after a successful and a failed transmission. ${ }^{5}$ Moreover, since the medium is declared as busy during the guard interval, to preserve traffic prioritization, type 1 nodes seize the channel after backoff, whereas type 2 nodes wait for additional $\Delta$ slots before decrementing backoff and attempting transmission.

Let us define the duration of a successful transmission as observed by nodes of type 1 if a node of type $y$ transmits $(y=1,2)$ as follows: $T_{s, y}=T_{h}+L_{y} / R+A I F S_{1}$, where $L_{y}$

\footnotetext{
${ }^{5}$ After a collision or in case of channel errors, $\Delta=\left(E I F S_{2}-\right.$ $\left.E I F S_{1}\right) / \sigma$, but due to EIFS settings [2], $\left(E I F S_{2}-E I F S_{1}\right)$ is equal to $\left(A I F S_{2}-A I F S_{1}\right)$
} 
is the length of type $y$ frames, $R$ is the data rate, ${ }^{6}$ and $T_{h}$ is the duration of the physical layer convergence protocol preamble and header. Analogously, let us define $T_{c, y}=T_{h}+$ $L_{y} / R+E I F S_{1}$ as the duration of a collision event (from the perspective of nodes of type 1) if only nodes of type $y$ transmit. The maximum duration of collision (from the perspective of nodes of type 1) if nodes of both types transmit is $T_{c, \max }=\max \left[T_{c, 1}, T_{c, 2}\right]$. The relevant durations of $T_{s, y}$, $T_{c, y}$, and $T_{c, \max }$ measured in slot times $(\sigma)$ are, respectively, as follows: $s_{y}=T_{s, y} / \sigma, c_{y}=T_{c, y} / \sigma$, and $c_{\max }=T_{c, \max } / \sigma$. It is enough to add $\Delta$ in the foregoing equations to get the duration of respective events (measured in slots) as they are observed by the nodes of type 2 .

For each type of nodes, we define $T_{y}$ as the useful duration of a CCH interval in slot times: $T_{y}=\left(T_{\mathrm{CCH}}-T_{g}-T_{h}-\right.$ $\left.L_{y} / R\right) / \sigma$, where $T_{\mathrm{CCH}}$ is the duration of $\mathrm{CCH}$ interval, and $T_{g}$ is the guard time. Indeed, frames cannot be transmitted during the guard time or if the residual time before the end of the $\mathrm{CCH}$ interval is shorter than the frame transmission time $\left(T_{h}+L_{y} / R\right)$.

The model aims to derive the successful frame delivery probability for both nodes of types 1 and 2, and to this purpose, we compute the probability of having $n_{1}$ nodes of type 1 and $n_{2}$ nodes of type 2 , with remaining windows $w_{1}$ and $w_{2}$, respectively, when there are $(l-1)$ empty slots, and there is a transmission in the $l$ th slot, $k_{y}$ nodes of type $y$ transmit, with $k_{y} \in\left[1, n_{y}\right]$ and $y=1,2$, assuming that at least one node from either type 1 or type 2 transmits, as follows:

$$
\begin{aligned}
P\left(l, n_{1}, n_{2}, w_{1}, w_{2}, k_{1}, k_{2}\right) & \left(1-\frac{l-1}{w_{1}}\right)^{n_{1}}\left(1-\frac{l-1}{w_{2}}\right)^{n_{2}} \\
= & \times\left(\begin{array}{c}
n_{1} \\
k_{1}
\end{array}\right)\left(\frac{1}{w_{1}-l+1}\right)^{k_{1}}\left(1-\frac{1}{w_{1}-l+1}\right)^{n_{1}-k_{1}} \\
& \times\left(\begin{array}{c}
n_{2} \\
k_{2}
\end{array}\right)\left(\frac{1}{w_{2}-l+1}\right)^{k_{2}}\left(1-\frac{1}{w_{2}-l+1}\right)^{n_{2}-k_{2}} .
\end{aligned}
$$

The foregoing probability is modeled through a Bernoulli process, where $1 /\left(w_{y}-l+1\right)$ is the probability of uniformly choosing any slot out of $\left(w_{y}-l+1\right)$ available slots $(y=1,2)$. If $k_{2}=0$ (first row) or $k_{1}=0$ (second row), then the preceding formula becomes

$$
\begin{aligned}
P\left(l, n_{1}, n_{2}, w_{1}, w_{2},\right. & \left.k_{1}, k_{2}\right) \\
= & \left\{\begin{array}{l}
\left(1-\frac{l-1}{w_{1}}\right)^{n_{1}}\left(1-\frac{l}{w_{2}}\right)^{n_{2}} \\
\quad \times\left(\begin{array}{c}
n_{1} \\
k_{1}
\end{array}\right)\left(\frac{1}{w_{1}-l+1}\right)^{k_{1}}\left(1-\frac{1}{w_{1}-l+1}\right)^{n_{1}-k_{1}} \\
\left(1-\frac{l-1}{w_{2}}\right)^{n_{2}}\left(1-\frac{l}{w_{1}}\right)^{n_{1}} \\
\times\left(\begin{array}{c}
n_{2} \\
k_{2}
\end{array}\right)\left(\frac{1}{w_{2}-l+1}\right)^{k_{2}}\left(1-\frac{1}{w_{2}-l+1}\right)^{n_{2}-k_{2}} .
\end{array}\right.
\end{aligned}
$$

The term $\left(1-l / w_{2}\right)$ when $k_{2}=0$ (the term $\left(1-l / w_{1}\right)$ when $\left.k_{1}=0\right)$ accounts for the probability that a node of type 2

\footnotetext{
${ }^{6}$ For ease of notation, we assume that both types of nodes use the same transmission data rate. Notwithstanding, the model can easily be extended to foresee transmissions of different types of frames at different data rates.
}

(type 1) does not choose one of the first $l$ slots to transmit, $(l-1)$ are empty slots, and the $l$ th slot is chosen by nodes of type 1 (type 2) to transmit.

In analogy to the model in [6], we define $P_{0}(l, n, w, k)$ as the probability that $n$ vehicles are in the system that select backoffs from a contention window of $w$ slots, $(l-1)$ empty slots pass before the first transmission attempt, and $k$ vehicles transmit in the $l$ th slot, i.e.,

$$
\begin{aligned}
P_{0}(l, n, w, k)=(1 & \left.-\frac{l-1}{w}\right)^{n}\left(\begin{array}{l}
n \\
k
\end{array}\right) \\
& \times\left(\frac{1}{w-l+1}\right)^{k}\left(1-\frac{1}{w-l+1}\right)^{n-k} .
\end{aligned}
$$

The mean number of successful transmissions $\widetilde{X}_{\text {success }}^{(1)}(t, w, n)$ if only $n$ nodes of type 1 with remaining windows $w$ are in the system and $t$ slots are left in $\mathrm{CCH}$ interval is computed as follows:

$$
\begin{aligned}
\widetilde{X}_{\text {success }}^{(1)}(t, w, n) & \\
=\sum_{l=1}^{\min (w, t)}\{ & P(l, n, w, 1)(1-\mathrm{BER})^{L_{1}} \\
& \times\left[1+\widetilde{X}_{\text {success }}^{(1)}\left(t-l+1-s_{1}, w-l, n-1\right)\right] \\
& +P(l, n, w, 1)\left(1-(1-\mathrm{BER})^{L_{1}}\right) \\
& \times \widetilde{X}_{\text {success }}^{(1)}\left(t-l+1-c_{1}, w-l, n-1\right) \\
& +\sum_{k=2}^{n} P(l, n, w, k) \widetilde{X}_{\text {success }}^{(1)} \\
& \left.\times\left(t-l+1-c_{1}, w-l, n-1\right)\right\} .
\end{aligned}
$$

The first term in this equation accounts for the probability that only one out of $n$ vehicles, choosing backoff from $w$ slots, successfully transmits in the $l$ th slot, and $\widetilde{X}_{\text {success }}^{(1)}(t-l+1-$ $\left.s_{1}, w-l, n-1\right)$ is the mean number of successful transmissions in the remaining $\left(t-l+1-s_{1}\right)$ slots; the second term corresponds to the case when the transmission is corrupted by noise, while the third term models $k(k \geq 2)$ of $n$ vehicles transmitting in the $l$ th slot and thus incurring collisions. Analogously, the mean number of successful transmissions $\widetilde{X}_{\text {success }}^{(2)}(t, w, n)$ if only nodes of type 2 are in the system is computed as follows:

$$
\begin{aligned}
\widetilde{X}_{\text {success }}^{(2)}(t, w, n) & \\
=\sum_{l=1}^{\min (w, t)}\{ & P(l, n, w, 1)(1-\mathrm{BER})^{L_{2}} \\
& \times\left[1+\widetilde{X}_{\text {success }}^{(2)}\left(t-l+1-\left(s_{1}+\Delta\right), w-l, n-1\right)\right] \\
& +P(l, n, w, 1)\left(1-(1-\mathrm{BER})^{L_{2}}\right) \\
& \times \widetilde{X}_{\text {success }}^{(2)}\left(t-l+1-\left(c_{1}+\Delta\right), w-l, n-1\right) \\
& +\sum_{k=2}^{n} P(l, n, w, k) \widetilde{X}_{\text {success }}^{(2)} \\
& \left.\times\left(t-l+1-\left(c_{1}+\Delta\right), w-l, n-1\right)\right\} .
\end{aligned}
$$


The main difference between equations for $\widetilde{X}_{\text {success }}^{(1)}(t, w, n)$ and $\widetilde{X}_{\text {success }}^{(2)}(t, w, n)$ lies in the fact that type 2 nodes have to wait for additional $\Delta$ slots before decrementing their backoff counter after a busy medium.

$\widetilde{X}_{\text {success }}^{(1)}\left(t, n_{1}, n_{2}, w_{1}, w_{2}\right)$ is a mean number of successful transmissions of type 1 frames during the $\mathrm{CCH}$ interval (given there are $t$ slots left, $n_{1}$ nodes of type 1 , and $n_{2}$ nodes of type 2 with $w_{1}$ and $w_{2}$ of their remaining contention windows, respectively), and $\Delta$ first slots are allocated for exclusive access of nodes of type 1, i.e.,

$$
\begin{aligned}
X_{\text {success }}^{(1)}\left(t, n_{1}, n_{2},\right. & \left.w_{1}, w_{2}\right) \\
& = \begin{cases}\widetilde{X}_{\text {success }}^{(1)}\left(t, n_{1}, w_{1}\right), & n_{2}=0 \\
0, & n_{1}=0 \\
* * *, & n_{1}>0, n_{2}>0\end{cases}
\end{aligned}
$$

where $* * *$ is defined at the bottom of the next page, and $P_{\Delta}\left(n_{1}, w_{1}\right)=\left\{\begin{array}{ll}\left(\frac{w_{1}-\Delta}{w_{1}}\right)^{n_{1}}, & \Delta<w_{1} \\ 0, & \Delta \geq w_{1}\end{array}\right.$ is the probability that no node of type 1 transmits in prioritized $\Delta$ slots.

The computation of $\widetilde{X}_{\text {success }}^{(1)}$ is recursive. ${ }^{7}$ The first case $\left(n_{2}=0\right)$ corresponds to the situation when there are only nodes of type 1 in the network, and therefore, the formula for $\widetilde{X}_{\text {success }}^{(1)}\left(t, n_{1}, w_{1}\right)$ is directly applied. The second case $\left(n_{1}=0\right)$ means that all nodes of type 1 have already performed their transmission attempts. The third case reflects the situation when both nodes of types 1 and 2 have frames to transmit. The formula for the third case represents a sum of two terms. The first term models the prioritized access of type 1 nodes during the first $\Delta$ slots after the channel was detected as busy. The second term comprises all possible situations occurring in the channel when both nodes of types 1 and 2 are contending for access. These situations are successful transmission of the node of type 1, error-prone transmission of the node of type 1, successful transmission of the node of type 2, error-prone transmission of the node of type 2, collision among nodes of type 1 , collision among nodes of type 2 , and, finally, collision among nodes of types 1 and 2 .

Analogously, the mean number of successful transmissions of type 2 frames during the $\mathrm{CCH}$ interval $X_{\text {success }}^{(2)}\left(T_{2}, N_{1}, N_{2}, W_{1}, W_{2}\right)$ can be derived. Then, the probability of success for nodes of type $y$, with $y=1,2$, can be computed as

$$
P_{\text {success }}^{(y)}=\frac{X_{\text {success }}^{(y)}\left(T_{1}, N_{1}, N_{2}, W_{1}, W_{2}\right)}{N_{y}} .
$$

The proposed analytical framework can be applied not only to compute the probabilities of successful transmission for the two types of frames but also to distinguish different reasons of frame loss: due to the noise in the channel, due to the collision, or due to lack of time in $\mathrm{CCH}$ interval. The latter event is due to the alternating WAVE channel switching and

\footnotetext{
${ }^{7}$ Due to recursive calculation, the computation time of the model depends on the number of considered nodes and other values of input parameters. Our experiments show that for many scenarios of interest, the model demonstrates reasonable computation time.
}

occurs when a node's backoff counter does not decrease to zero before the end of a CCH interval, and therefore, the frame is dropped to avoid the transmission of outdated information in the subsequent $\mathrm{CCH}$ interval. Generalized formulas for all these cases are presented in the Appendix.

\section{Performance Evaluation}

\section{A. Simulation Settings}

An event-driven custom simulation program has been developed in Matlab that accurately implements the system behavior under the simplifying model assumptions. All simulated vehicular nodes are static; they are within reciprocal communication range and have one new broadcast frame, either WSA or beacon, ready to transmit at the beginning of each $\mathrm{CCH}$ interval.

Without loss of generality, WSAs are considered type 1 frames and beacons type 2 frames. During simulations, the number of type 2 nodes $\left(N_{2}\right)$ that transmit beacons changes from 1 to 50, whereas the number of type 1 nodes $\left(N_{1}\right)$ acting as WAVE providers and sending WSA frames is set to 1 or 5 . The example with one provider $\left(N_{1}=1\right)$ represents the case of an RSU offering services to nearby vehicles, whereas in the second case $\left(N_{1}=5\right)$, the provider role could be played by OBUs.

The simulator closely follows the 802.11p MAC protocol specifications for each independently transmitting node by accounting for CSMA/CA rules, EDCA prioritization, WAVE multichannel operations, and related phenomena.

At the beginning of the $\mathrm{CCH}$ interval, after the guard time, each station independently selects a random backoff delay in the range $[0, C W \min [A C]]$ and starts the countdown while sensing the channel slot by slot. Transmission fails if the backoff of more stations simultaneously reaches zero (collision), or if the transmitted frame is affected by errors. Transmission also fails if the backoff counter reaches zero when the residual $\mathrm{CCH}$ interval is not sufficient to accommodate the frame transmission or if the backoff counter does not reach zero during the $\mathrm{CCH}$ interval; in the latter cases, the packet is dropped at the sender side.

The first set of simulations aims to validate the proposed novel analytical approach and to evaluate effectiveness of the access parameter sets suggested by IEEE [3] and DSRC [12] standard documents under different channel and traffic load conditions.

The second set of results aims to clearly characterize the negative influences of channel switching on the vehicular network performance and to bring some insight into possible improvements that can be achieved by moving away from standard suggestions.

MAC parameters in common to both sets of results are as follows: aSlotTime $=16 \mu \mathrm{s}$, aSIFSTime $=30 \mu \mathrm{s}$, and header duration $\left(T_{h}\right)=40 \mu \mathrm{s}$.

\section{B. Performance With Standardized Parameters}

With regard to AC assignment to WSAs, we follow the IEEE 1609.4 indications [3] to encapsulate WSAs in 802.11 management frames taking the highest access priority (AC_VO) as well as the DSRC indications [12] that suggest AC_BE 
TABLE II

ACs Suggested by Standards For WSA And Beacon Frames

\begin{tabular}{lccc}
\hline \hline $\begin{array}{c}\text { Access } \\
\text { category }\end{array}$ & Frame Type & CWmin[AC] & AIFSN[AC] \\
\hline$A C_{-} B E$ & Beacon, WSA & 15 & 6 \\
$A C_{-} V I$ & Beacon & 7 & 3 \\
$A C_{-} V O$ & WSA & 3 & 2 \\
\hline \hline
\end{tabular}

for WSA frames. Regarding beacons, we refer to AC_VI and AC_BE ACs, as proposed by DSRC in [12] for heartbeat messages. With the given settings, the simulated AC parameters for types 1 and 2 frames are reported in Table II.
In summary, four possible $\mathrm{AC}$ combinations are suggested by the standards: 1) AC_VO for WSAs and AC_VI for beacons; 2) AC_VO for WSAs and AC_BE for beacons; 3) AC_BE for WSAs and AC_VI for beacons ${ }^{8}$; and 4) AC_BE for both WSA and beacon frames.

The specific values of AIFSN and $C W$ min for AC combinations 1) and 3) may lead to inter-class collisions between frames of different ACs due to overlapping backoffs, as shown in Fig. 3(a). Inter-class collisions occur when the backoff

${ }^{8}$ It should be noticed that in this case, the priorities of beacons and WSAs are inverted.

$$
\begin{aligned}
& * * *=\sum_{l=1}^{\min \left(t, \Delta, w_{1}\right)}\left\{P_{0}\left(l_{1}, n_{1}, w_{1}, 1\right)(1-\mathrm{BER})^{L_{1}}\right. \\
& \times\left[1+X_{\text {success }}^{(1)}\left(t-l+1-s_{1}, n_{1}-1, n_{2}, w_{1}-l, w_{2}\right)\right] \\
& +P_{0}\left(l, n_{1}, w_{1}, 1\right)\left(1-(1-\mathrm{BER})^{L_{1}}\right) X_{\text {success }}^{(1)} \\
& \times\left(t-l,+1-c_{1}, n_{1}-1, n_{2}, w_{1}-l, w_{2}\right) \\
& +\sum_{k=2}^{n_{1}} P_{0}\left(l, n_{1}, w_{1}, k\right) X_{\text {success }}^{(1)} \\
& \left.\times\left(t-l,+1-c_{1}, n_{1}-k, n_{2}, w_{1}-l, w_{2}\right)\right\} \\
& +P_{\Delta}\left(n_{1}, w_{1}\right) \sum_{l=1}^{\min \left(w_{1}-\Delta, t-\Delta\right)}\left\{P\left(l, n_{1}, n_{2}, w_{1}-\Delta, w_{2}, 1,0\right)(1-\mathrm{BER})^{L_{1}}\right. \\
& \times\left[1+X_{\text {success }}^{(1)}\left(t-l+1-s_{1}-\Delta, n_{1}-1, n_{2}, w_{1}-l-\Delta, w_{2}-l\right)\right] \\
& +P\left(l, n_{1}, n_{2}, w_{1}-\Delta, w_{2}, 1,0\right)\left(1-(1-\mathrm{BER})^{L_{1}}\right) \\
& \times X_{\text {success }}^{(1)}\left(t-l+1-c_{1}-\Delta, n_{1}-1, n_{2}, w_{1}-l-\Delta, w_{2}-l\right) \\
& +P\left(l, n_{1}, n_{2}, w_{1}-\Delta, w_{2}, 0,1\right)(1-\mathrm{BER})^{L_{2}} \\
& \times X_{\text {success }}^{(1)}\left(t-l+1-s_{2}-\Delta, n_{1}, n_{2}-1, w_{1}-l-\Delta, w_{2}-l\right) \\
& +P\left(l, n_{1}, n_{2}, w_{1}-\Delta, w_{2}, 0,1\right)\left(1-(1-\mathrm{BER})^{L_{2}}\right) \\
& \times X_{\text {success }}^{(1)}\left(t-l+1-c_{2}-\Delta, n_{1}, n_{2}-1, w_{1}-l-\Delta, w_{2}-l\right) \\
& +\sum_{k_{1}=2}^{n_{1}}\left[P\left(l, n_{1}, n_{2}, w_{1}-\Delta, w_{2}, k_{1}, 0\right)\right. \\
& \left.\times X_{\text {success }}^{(1)}\left(t-l+1-c_{1}-\Delta, n_{1}-k_{1}, n_{2}, w_{1}-l-\Delta, w_{2}-l\right)\right] \\
& +\sum_{k_{2}=2}^{n_{2}}\left[P\left(l, n_{1}, n_{2}, w_{1}-\Delta, w_{2}, 0, k_{2}\right)\right. \\
& \left.\times X_{\text {success }}^{(1)}\left(t-l+1-c_{2}-\Delta, n_{1}, n_{2}-k_{2}, w_{1}-l-\Delta, w_{2}-l\right)\right] \\
& +\sum_{k_{1}=1}^{n_{1}} \sum_{k_{2}=1}^{n_{2}}\left[P\left(l, n_{1}, n_{2}, w_{1}-\Delta, w_{2}, k_{1}, k_{2}\right)\right. \\
& \times X_{\text {success }}^{(1)}\left(t-l+1-c_{\max }-\Delta, n_{1}-k_{1},\right. \\
& \left.\left.\left.n_{2}-k_{2}, w_{1}-l-\Delta, w_{2}-l\right)\right]\right\}
\end{aligned}
$$


$\operatorname{AIFSN}_{1}=2, \mathrm{CWmin}_{1}=3$ $\operatorname{AIFSN}_{2}=3, \mathrm{CWmin}_{2}=7$ $\Delta=\operatorname{AIFSN}_{2}-$ AIFSN $_{1}=1$

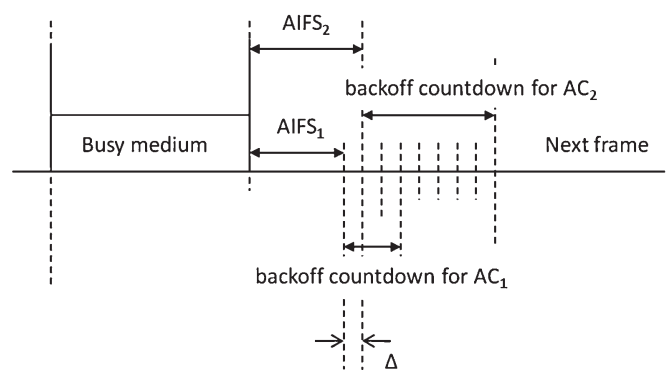

(a)

$$
\begin{aligned}
& \text { AIFSN }_{1}=2, \mathrm{CWmin}_{1}=3 \\
& \mathrm{AIFSN}_{2}=6, \mathrm{CWmin}_{2}=15 \\
& \Delta=\mathrm{AIFSN}_{2}-\mathrm{AIFSN}_{1}=4
\end{aligned}
$$

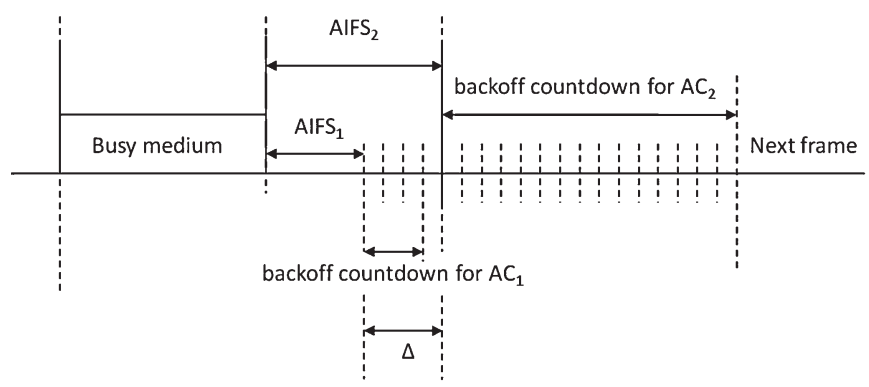

(b)

Fig. 3. (a) AC_VO for WSAs and AC_VI for beacons $\left(\Delta<W_{1}\right)$ : overlapping backoffs. (b) AC_VO for WSAs and AC_BE for beacons $\left(\Delta \geq W_{1}\right)$ : non-overlapping backoffs.

countdowns for types 1 and 2 frames expire simultaneously. AC combination 2), on the contrary, is immune to inter-class collisions thanks to no overlapping backoffs, as shown in Fig. 3(b), and only suffers from intra-class collisions among frames of the same type. Combination 4) is a special case when types 1 and 2 frames contend with the same access parameters.

To validate the analytical model, we compute the successful delivery probability of type $1 P_{\text {success }}^{(1)}$ and type 2 frames $P_{\text {success }}^{(2)}$, henceforth respectively shortened as $P_{1}$ and $P_{2}$, when varying the number of nodes $N_{1}$ (1 or 5 ) and $N_{2}$ (from 1 to 10$)$, under ideal $(\mathrm{BER}=0)$ and error-prone $\left(\mathrm{BER}=10^{-4}\right)$ channel status, and when fixing the data rate to $3 \mathrm{Mb} / \mathrm{s}$ for the most robust transmission scheme. Validation results are plotted in Figs. 4 and 5 for AC combinations 1) and 2), respectively. Analytical results (lines) closely match the simulation results (symbols), thus witnessing the accuracy and precision of the proposed model. Indeed, the analytical approach does not involve any approximation and exactly describes the stochastic process representing independent operations of two types of nodes for any possible set of input parameter values.

With regard to the effectiveness and robustness of the AC combinations 1) and 2) proposed by the standards, results are not encouraging. Figs. 4 and 5 show that the WSA and beacon delivery probabilities, $P_{1}$ and $P_{2}$, respectively, are adversely affected by an increasing number of nodes.

Specifically, the beacon performance $P_{2}$ is insensitive to $N_{1}$ (the two curves for $N_{1}=1$ and $N_{1}=5$ coincide), but it rapidly decreases when $N_{2}$ increases due to more collisions among a

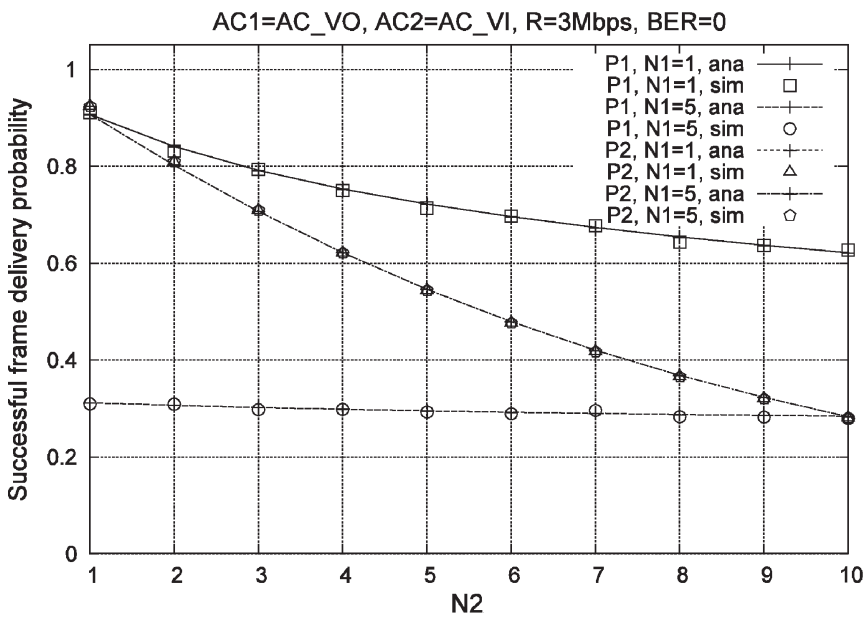

(a)

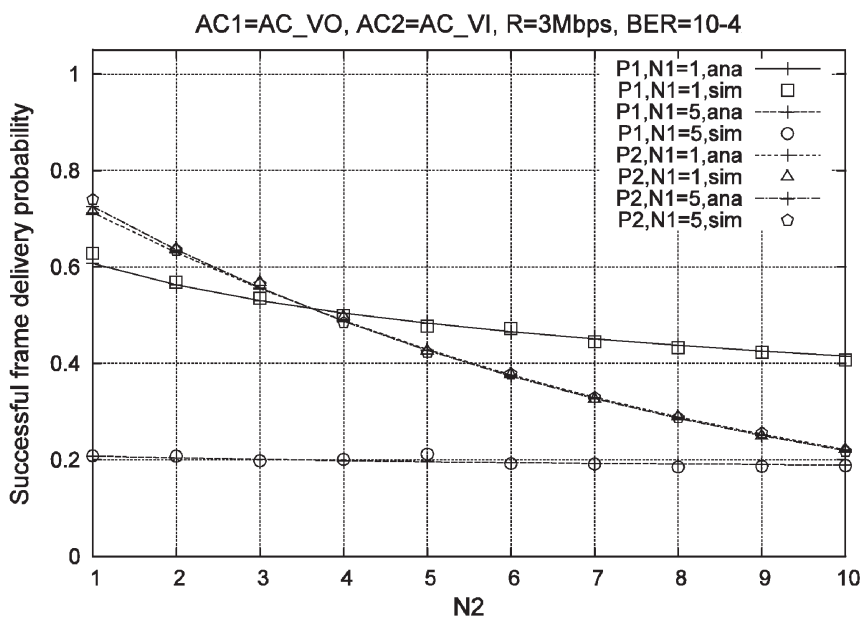

(b)

Fig. 4. Successful delivery probability versus the number of type 2 nodes sending beacons. Overlapping backoffs, AC combination 1). (a) BER $=0$ (b) $\mathrm{BER}=10^{-4}$.

higher number of vehicles. The invariance of $P_{2}$ with $N_{1}$ can intuitively be explained for AC combination 2) in Fig. 5.

It is due to the specific AC settings that imply nonoverlapping backoffs, so only intra-class collisions can occur, and the beacon delivery probability $P_{2}$ only depends on the number of contending vehicles $N_{2}$ in the same class. For AC combination 1) in Fig. 4, instead, the insensitiveness of $P_{2}$ to $N_{1}$ has a different motivation. Even if the $\mathrm{AC}$ parameter settings, corresponding to the overlapping backoff case, could potentially create inter-class collisions, nevertheless, the low number of nodes of type 1 and the large contention window of type 2 nodes make the occurrence of such events negligible. Thereby, type 2 frame delivery is more adversely affected by intra-class collisions than by inter-class collisions.

It can also be observed that when using $\mathrm{AC}$ combination 2) instead of combination 1), the use of AC_BE for type 2 frames (in Fig. 5) with a larger $C W$ size, as compared with $\mathrm{AC}_{-} \mathrm{VI}$ (in Fig. 4), helps in increasing the beacon delivery probability $P_{2}$.

The WSA delivery probability $P_{1}$ decreases when $N_{1}$ increases due to an increase in intra-class collisions. The trend of $P_{1}$ when $N_{2}$ increases is different in Figs. 4 and 5. For the non-overlapping backoff case of AC combination 2) in Fig. 5, 


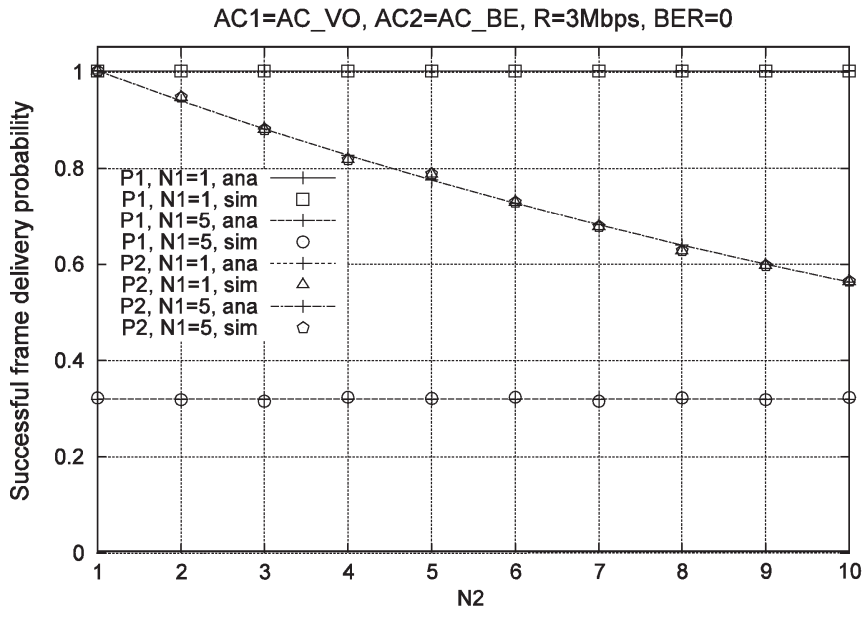

(a)

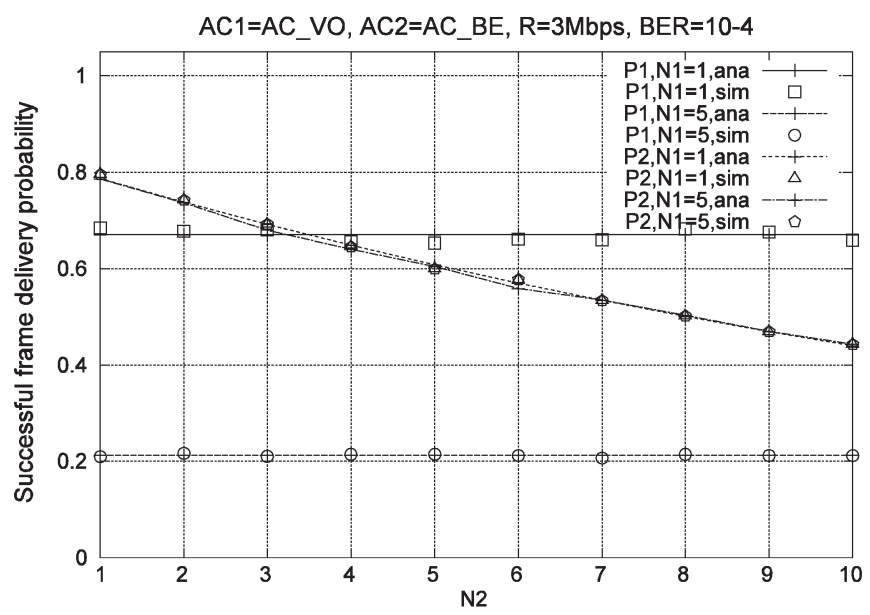

(b)

Fig. 5. Successful delivery probability versus the number of type 2 nodes sending beacons. Non-overlapping backoffs, AC combination 2). (b) AC_BE; $\mathrm{BER}=0$. (b) $\mathrm{BER}=10^{-4}$.

$P_{1}$ takes constant values, regardless of the number of nodes of type 2 . When $N_{1}=1$ and $\mathrm{BER}=0, P_{1}$ is constantly equal to one, because the unique provider in the system always transmits successfully. This value decreases to about 0.7 for $\mathrm{BER}=10^{-4}$ due to losses induced by channel errors. When $N_{1}=5$, due to the higher number of nodes in the same class contending with a small contention window $\left(C W \min _{1}=3\right)$, collisions increase, and $P_{1}$ significantly decreases (down to a value lower than 0.4 when $\mathrm{BER}=0$, or 0.2 when $\mathrm{BER}=10^{-4}$ ). These results show that when increasing the number of type 1 nodes, the effect of WSA traffic prioritization is cancelled due to the adverse effect of intra-class collisions that are exacerbated by the use of a small contention window.

For the overlapping backoff case of AC combination 1) in Fig. 4, an increase in $N_{2}$ is more detrimental on $P_{1}$ for the case with one provider. The probability of inter-class collisions increases with $N_{2}$, and the unique type 1 node in the system (which selects its backoff from a small $C W$ size) will be more often prevented to access the channel due to competition with many nodes of type 2 . For the case with five providers, $P_{1}$ is insensitive to $N_{2}$ due to the predominant effect of intraclass collisions over inter-class collisions that also, in this case, nullify the traffic prioritization effect.

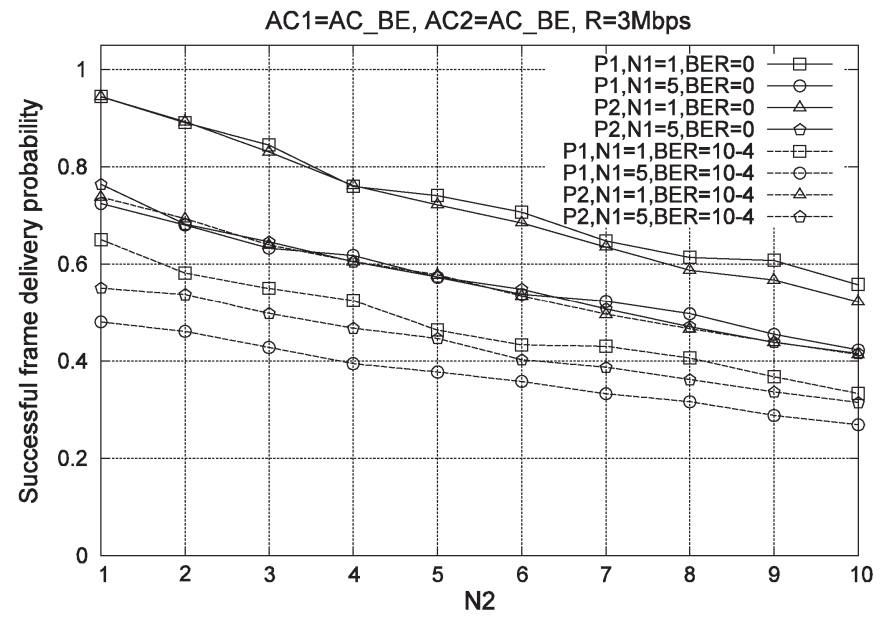

Fig. 6. Successful delivery probability versus the number of type 2 nodes sending beacons AC combination 4).

The adverse effect of BER is evident in Figs. 4 and 5 and results in a significant reduction of both $P_{1}$ and $P_{2}$. The effect is, however, more detrimental on the performance of type 1 frames; this is due to the longer frame size that makes WSAs $(500 \mathrm{~B})$ more vulnerable to channel errors compared with beacons (300 B).

The unsatisfactory delivery performance in Figs. 4 and 5 when $N_{1}$ and $N_{2}$ increase asks for different AC settings. A first straightforward solution to improve the WSA frame delivery could be the use of larger $C W \mathrm{~s}$, hence shifting WSAs to a lower priority AC. We repeated the previous study when considering $\mathrm{AC}$ combinations 3 ) and 4) with WSAs belonging to $\mathrm{AC} \_\mathrm{BE}$ and beacons to $\mathrm{AC} \_\mathrm{VI}$ and $\mathrm{AC} \_\mathrm{BE}$, respectively. Results for combination 3) are not shown, since in this case as well, as for combination 1), the overlapping backoffs have detrimental effects on the performances of the traffic class using the smaller window size (in this case, even if the WSA delivery probability increases, the beacon delivery probability becomes unacceptable).

Fig. 6 reports the results achieved for combination 4) when both WSAs and beacons are assigned $\mathrm{AC}_{-} \mathrm{BE}^{9}$. In this case, type 1 nodes contend with type 2 nodes to access the medium with the same parameters set; therefore, they equally share the available capacity. As a result, under ideal channel conditions, $P_{1}$ almost coincides with $P_{2}$ in the plot. When BER is considered instead, $P_{1}$ is lower than $P_{2}$ because of the larger WSA frame size.

The use of a larger contention window for type 1 frames has the positive effect of reducing intra-class collisions compared with AC combinations 1) and 2) for the case with five providers, and therefore, $P_{1}$ increases in this case. This is achieved at the price of a slight decrease in $P_{2}$ as compared with AC combinations 1) and 2) due to competition with type 1 nodes that now belong to the same AC as type 2 nodes. Improvements are not achieved for $P_{1}$ when only one provider exists due to the contention of this provider with all the other nodes of type 2 belonging to the same AC.

\footnotetext{
${ }^{9} \mathrm{We}$ did not present analytical results for this scenario to reduce cluttering of Fig. 6 ,
} 
In summary, these findings suggest that in the realistic case of having more than one provider in a given area, the use of a low-priority AC with a large contention window should be recommended to combat intra-class collisions more effectively. Therefore, under typical traffic conditions, i.e., more providers sending WSAs and many vehicles transmitting beacons, the highest priority ACs should be avoided to reduce intra-class collisions, and large contention windows should be used. This is why, in the remainder of this paper, we continue to analyze the performance of combination 4) that achieves the best tradeoff between the successful delivery probability of both types of nodes.

Notwithstanding, even with this AC setting, an increase in $\mathrm{N}_{2}$ could cause significant performance degradation. This is mainly due to the fact that since all nodes extract their backoff at the beginning of the $\mathrm{CCH}$ interval, even with the largest $C W$ suggested by the standard, i.e., 15 , frame collisions are very likely to occur when the number of nodes becomes large.

\section{Combating the Negative Influence of Channel Switching}

Here, we take as a starting point the AC settings suggested by the standards providing the best tradeoff in typical traffic conditions, that is, combination 4) with both WSAs and beacons belonging to AC_BE, and we identify the key parameters affecting the channel-switching-induced phenomena to bring some insight into ways to combat them and give hints on the parameter usage/settings for possible performance enhancement. We analyze the effects of different frame lengths $L^{10}(100,500,1000,1400 \mathrm{~B}), C W$ size, and total number of nodes $N=\left(N_{1}+N_{2}\right)$ under ideal and error-prone channel conditions. Results in the previous section show that also AC combination 4) could fail in achieving acceptable frame delivery when the traffic load increases, mainly due to synchronized frame collisions at the beginning of $\mathrm{CCH}$ interval. The main straightforward solution to counteract the latter event is to increase $C W$ further to achieve higher time diversity in the transmission attempts.

In Fig. 7, the frame loss probability, which is computed as $\left(1-P_{i}\right)$ for type $i$ frames ${ }^{11}$, is depicted when increasing the $C W$ size up to 511 , that is, the maximum value allowed by $802.11 \mathrm{p}$, when $N=50$ and the data rate is $3 \mathrm{Mb} / \mathrm{s}$. An increase in $C W$ has a positive effect in any case; however, for shorter frames, the gain is more remarkable than for longer frames. For very short frame sizes, i.e., $L=100 \mathrm{~B}$, the loss probability monotonically decreases when increasing $C W$. For any $L$ value greater than $100 \mathrm{~B}$, the loss probability decreases until the $C W$ size reaches 255; then surprisingly, a further increase in $C W$ does not help in decreasing the frame loss probability. In fact, a large window, on the one hand, reduces collisions and frame losses, but on the other hand, it is more prone to losses due to $\mathrm{CCH}$ interval expiry.

As $L$ increases, the negative impact of BER on frame loss probability also becomes more remarkable. The effects of dif-

\footnotetext{
${ }^{10}$ Without loss of generality, henceforth, we assume that WSAs and beacons have the same frame size $L_{1}=L_{2}=L$

${ }^{11}$ Since the same AC is used for the two types of frames, the results for types 1 and 2 frames coincide.
}

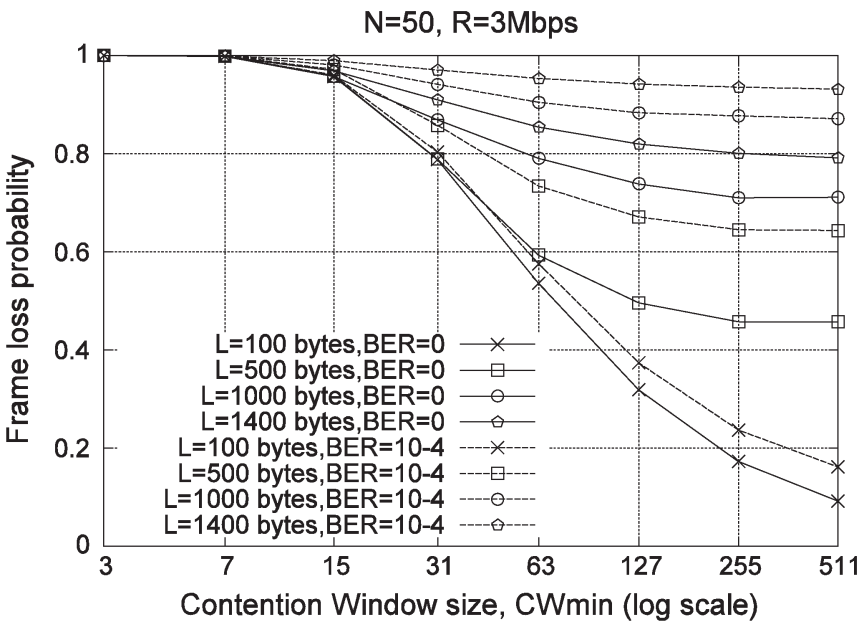

Fig. 7. Overall loss probability versus minimum $\mathrm{CW}$ size for different frame lengths $L$.

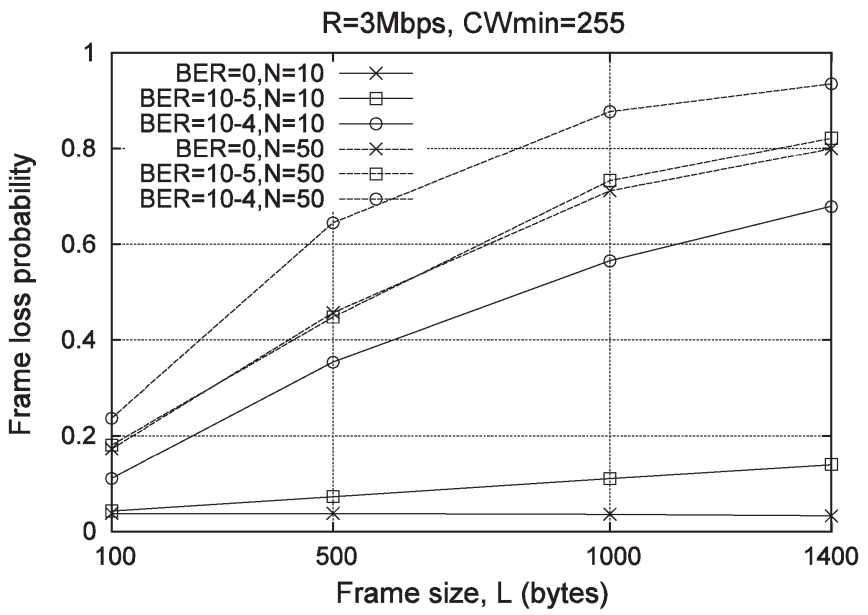

Fig. 8. Overall loss probability versus frame length $L$ under different BER and load conditions.

ferent BER values $\left(0,10^{-4}, 10^{-5}\right)$ and different frame sizes $L$ on the frame loss probability can be observed in Fig. 8 under a variable number of nodes $N(10,50)$ and for a fixed $C W$ equal to 255 . At low traffic load $(N=10)$, under ideal channel conditions $(\mathrm{BER}=0)$, a very low and constant loss probability is experienced whatever the frame size; under errorprone channel conditions, instead, the frame loss significantly increases with the frame size $L$, and very poor performance is achieved when increasing the BER. When focusing on high traffic load conditions $(N=50)$, no remarkable differences are noticed when passing from BER $=0$ to BER $=10^{-5}$ since the main loss contributions come from collisions and $\mathrm{CCH}$ interval expire time. Unacceptable frame loss probability is experienced at higher than 0.8 , and performance becomes worse for $\mathrm{BER}=10^{-4}$

In the histograms in Figs. 9 and 10, different causes of frame loss are clearly identified: collisions (labeled as $C O L$ ), $\mathrm{CCH}$ interval expiry (labeled as $E X P$ ), and channel errors (labeled as BER). Results are achieved for a variable number of nodes $N$ (ranging from 10 to 50 ), a fixed frame size $L$ of $500 \mathrm{~B}$, and two $C W$ sizes equal to 15 and 255 , which are the two 


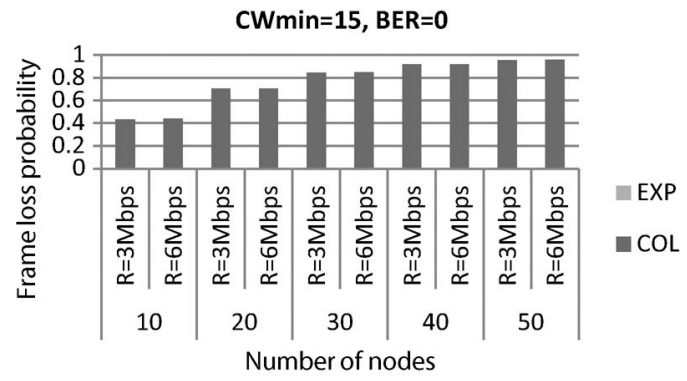

(a)

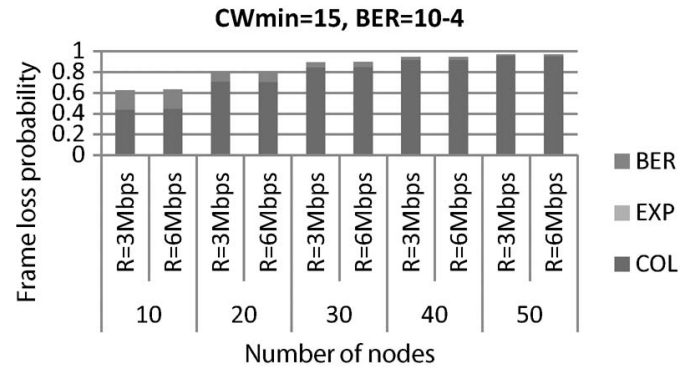

(b)

Fig. 9. Overall loss probability versus number of nodes for $\mathrm{CWmin}=15$, $L=500 \mathrm{~B}$, for (a) BER $=0$ and (b) $\mathrm{BER}=10^{-4}$.

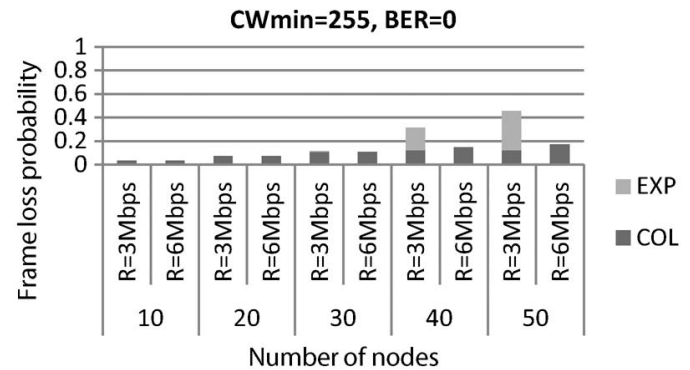

(a)

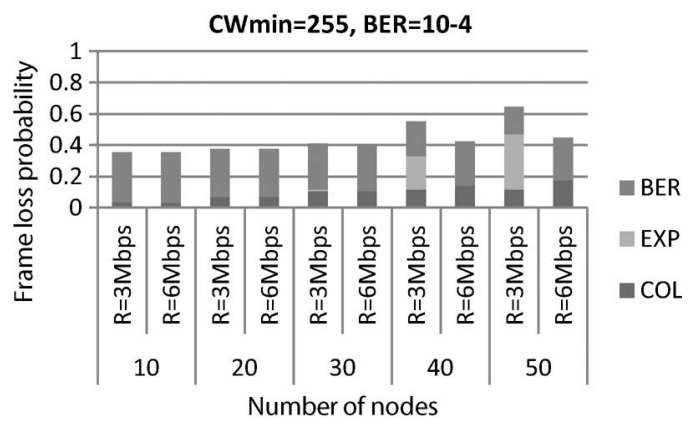

(b)

Fig. 10. Overall loss probability versus number of nodes for $\mathrm{CWmin}=255$, $L=500 \mathrm{~B}$, for (a) BER $=0$ and (b) $\mathrm{BER}=10^{-4}$.

most robust transmission modes with a data rate $R$ of $3 \mathrm{Mb} / \mathrm{s}$ and $6 \mathrm{Mb} / \mathrm{s}$, under ideal and error-prone channel. The results in Fig. 9(a) show that for $C W$ equal to 15, in the absence of channel errors, frames are only lost because of collisions at any traffic load. Naturally, the frame loss probability due to collisions increases with the number of nodes. Even under error-prone channel conditions, in Fig. 9(b), collisions represent the strongest contribution to frame loss, while the contribution of losses due to BER decreases as the traffic load increases.
TABLE III

SUMMARIZED FINDINGS

\begin{tabular}{ccccc}
\hline \hline $\begin{array}{c}\text { Access } \\
\text { category }\end{array}$ & TOT & COL & EXP & BER \\
\hline$W$ & $\downarrow$ & $\downarrow$ & $\uparrow$ & - \\
$N$ & $\uparrow$ & $\uparrow$ & $\uparrow$ & - \\
$R$ & $\downarrow$ & - & $\downarrow$ & - \\
$L$ & $\uparrow$ & - & $\uparrow$ & $\uparrow$ \\
\hline \hline
\end{tabular}

The use of a larger $C W$ equal to 255 in Fig. 10 instead makes the frame loss due to $\mathrm{CCH}$ interval expiry to represent the strongest contribution to the overall loss. Indeed, for $R=$ 3 Mbps and $N=50$, more than $50 \%$ of the frame losses are due to $\mathrm{CCH}$ expiry for both BER $=0$ and BER $=10^{-4}$.

When considering the effect of data rate $R$ in Figs. 9 and 10, again, it is possible to observe different trends. With a small $C W$ (15 in Fig. 9), there is no advantage in increasing the data rate from 3 to $6 \mathrm{Mb} / \mathrm{s}$, since frame losses are only due to collisions or BER, and there are no losses due to $\mathrm{CCH}$ expiry time. When increasing the $C W$ size to 255 in Fig. 10, for a total number of nodes lower than or equal to 30 , there is no advantage in increasing the data rate from 3 to $6 \mathrm{Mb} / \mathrm{s}$. When further increasing the number of nodes ( $N$ equal to 40 and 50), instead, at a higher data rate, it is very effective in reducing the overall frame losses, particularly acting on the losses due to $\mathrm{CCH}$ expiring time, since the frame transmission time at $6 \mathrm{Mb} / \mathrm{s}$ is shorter than at $3 \mathrm{Mb} / \mathrm{s}$.

For the reader's convenience, in Table III, we summarize the main findings of the conducted analysis. It shows how an increase of a given parameter, i.e., the contention window size $(C W)$, the number of nodes $(N)$, the data rate $(R)$, and the frame size $(L)$, can affect the overall frame loss probability (shortened as TOT) and the single loss contributions $(C O L$, $E X P$, and BER). Up/down arrows respectively indicate increasing/decreasing trend for the considered loss, and a dash indicates that the parameter does not influence the frame loss.

\section{Discussion AND CONCLUSION}

In this paper, we have presented a novel analytical framework for modeling broadcast traffic prioritization on the $\mathrm{CCH}$ of an 802.11p/WAVE vehicular network with the aim of understanding the potentialities and limits of this timely technology. The focus has been on periodic broadcast messages, like beacons and WSAs, which are expected to be frequently and massively transmitted in a vehicular network under normal operating conditions, unlike event-based safety-critical messages that are transmitted only in the case of an emergency.

While the $802.11 \mathrm{p}$ standard clearly establishes that safety messages have the highest access priority, it does not specify mandatory AC assignment for the other types of broadcast traffic under consideration in this paper. We have, therefore, analyzed the effects of different EDCA parameter sets (AC with relevant contention window and AIFS) on network performance under various traffic load, error-prone channel, frame sizes, and transmission data rates.

All the model simplifying assumptions (e.g., simple error channel model, no hidden terminals) are in common with 
up-to-date analytical studies of multiple access protocols for vehicular networks in the scientific literature, but in contrast to them, our model focuses on the prioritization of broadcast traffic and takes into account the multichannel operation of the 802.11p/WAVE standard with related periodic and synchronous channel switching.

The proposed analytical approach precisely models the underlying multidimensional stochastic process and accurately matches simulations results; it proved to be a valuable tool for the study of vehicular network performances and represents one more step toward the realistic mathematical modeling of 802.11p/WAVE.

Model validation has been completed with a wide simulation campaign that accounts of the latest 802.11p and 1609 standard versions. Results have shown that the assignment of ACs suggested in standard documents is not always satisfactory. The main reason is the use of a short contention window, which is kept limited even in the case of the lowest suggested $\mathrm{AC}$ (i.e., best effort with $C W=15$ ). Small windows exacerbate intra-class collisions that significantly affect performance degradation.

The straightforward solution to counteract the detrimental effects of synchronized frame collisions at the beginning of the $\mathrm{CCH}$ interval is to increase the contention window size to achieve a better time diversity in transmission attempts and reduce collisions. Results have shown that increasing the window size is successful in terms of reduction in frame loss probability. Nevertheless, larger contention windows are more prone to frame losses due to backoff counters that do not expire before the end of a $\mathrm{CCH}$ interval; this puts a limit on the window size. Another variable that can counteract these losses is the transmission data rate; it could be increased, but the increase should be kept limited $(6 \mathrm{Mb} / \mathrm{s}$ seems to be a good choice) when considering that under error-prone channels higher data rates are more vulnerable to interference. Finally, the use of shorter frames could be recommended to reduce both the impact of channel-induced errors and losses due to the expiration of the $\mathrm{CCH}$ interval, but while taking in mind that the frame size cannot be decreased unless to renounce to some conveyed information (e.g., security overhead, as suggested in [22]).
Analytical and simulative investigations of enhanced transmission techniques aimed to improve transmission reliability will be a subject matter for future work.

\section{APPENDIX}

Here, we provide generalization of the proposed analytical approach aiming to compute the probabilities of different events related to frame loss. The mean number of events if there are only $n$ nodes of type $i$ (either 1 or 2 ) with remaining contention windows $w$ are in the system and $t$ slots are left in $\mathrm{CCH}$ interval is denoted as $\widetilde{X}_{\text {event }}^{(i)}(t, w, n)$. Possible events are success, noise (frame is distorted by noise), and collision. The equation for $\tilde{X}_{\text {event }}^{(i)}(t, w, n)$, shown at the bottom of the page, holds.

Explanation of the notations: $C_{\text {event }}^{(i)}$ is equal to 1 if the equation for the event related to nodes of type $i$ is considered and is equal to 0 otherwise; $\Delta_{i}$ is equal to $\Delta$ if $i$ is 2 and is equal to 0 otherwise.

Analogously, let $X_{\text {event }}^{(i)}\left(t, n_{1}, n_{2}, w_{1}, w_{2}\right)$ be the mean number of events for type $i$ frames during the $\mathrm{CCH}$ interval (given there are $t$ slots left, there are $n_{1}$ nodes of type 1 and $n_{2}$ nodes of type 2 with $w_{1}$ and $w_{2}$ as their remaining contention windows, respectively)

$$
\begin{aligned}
& X_{\text {event }}^{(i)}\left(t, n_{1}, n_{2}, w_{1}, w_{2}\right) \\
& = \begin{cases}\tilde{X}_{\text {event }}^{(i)}\left(t, n_{i}, w_{i}\right), & n_{\bar{\iota}}=0 \\
0, & n_{i}=0 \\
* * *, & n_{i}>0, n_{\bar{\iota}}>0\end{cases}
\end{aligned}
$$

where $\left({ }^{* *}\right)$ is defined at the top of the next page, $\iota$ is equal to 1 if $i=2$, and $i$ is equal to 2 if $i=1$. Hence, the probabilities of the corresponding events are

$$
P_{\text {event }}^{(i)}=\frac{X_{\text {event }}^{(i)}\left(T_{i}, N_{1}, N_{2}, W_{1}, W_{2}\right)}{N_{i}}
$$

and the probability of the frame of type $i$ drop due to the lack of time on $\mathrm{CCH}$ interval is computed as

$$
P_{\text {residual }}^{(i)}=1-P_{\text {success }}^{(i)}-P_{\text {collision }}^{(i)}-P_{\text {noise }}^{(i)} .
$$

$$
\begin{aligned}
\tilde{X}_{\text {event }}^{(i)}(t, w, n)=\sum_{l=1}^{\min (w, t)}\{ & P(l, n, w, 1)(1-\mathrm{BER})^{L_{i}} \\
& \times\left[C_{\text {success }}^{(i)}+\tilde{X}_{\text {event }}^{(i)}\left(t-l+1-\left(s_{1}+\Delta_{i}\right), w-l, n-1\right)\right] \\
& +P(l, n, w, 1)\left(1-(1-\mathrm{BER})^{L_{i}}\right) \\
& \times\left[C_{\text {noise }}^{(i)}+\tilde{X}_{\text {event }}^{(i)}\left(t-l+1-\left(c_{1}+\Delta_{i}\right), w-l, n-1\right)\right] \\
& \left.+\sum_{k=2}^{n} P(l, n, w, k)\left[C_{\text {collision }}^{(i)}+\tilde{X}_{\text {event }}^{(i)}\left(t-l+1-\left(c_{1}+\Delta_{i}\right), w-l, n-k\right)\right]\right\}
\end{aligned}
$$




$$
\begin{aligned}
& * * *=\sum_{l=1}^{\min \left(t, \Delta, w_{1}\right)}\left\{P_{0}\left(l_{1}, n_{1}, w_{1}, 1\right)(1-\mathrm{BER})^{L_{1}}\left[C_{\text {success }}^{(1)}+X_{\text {event }}^{(1)}\left(t-l+1-s_{1}, n_{1}-1, n_{2}, w_{1}-l, w_{2}\right)\right]\right. \\
& +P_{0}\left(l, n_{1}, w_{1}, 1\right)\left(1-(1-\mathrm{BER})^{L_{1}}\right)\left[C_{\text {noise }}^{(1)}+X_{\text {event }}^{(1)}\left(t-l,+1-c_{1}, n_{1}-1, n_{2}, w_{1}-l, w_{2}\right)\right] \\
& \left.+\sum_{k=2}^{n_{1}} P_{0}\left(l, n_{1}, w_{1}, k\right)\left[C_{\text {collision }}^{(1)}+X_{\text {event }}^{(i)}\left(t-l,+1-c_{1}, n_{1}-k, n_{2}, w_{1}-l, w_{2}\right)\right]\right\} \\
& +P_{\Delta}\left(n_{1}, w_{1}\right) \sum_{l=1}^{\min \left(w_{1}-\Delta, t-\Delta\right)}\left\{P\left(l, n_{1}, n_{2}, w_{1}-\Delta, w_{2}, 1,0\right)(1-\mathrm{BER})^{L_{1}}\right. \\
& \times\left[C_{\text {success }}^{(1)}+X_{\text {event }}^{(i)}\left(t-l+1-s_{1}-\Delta, n_{1}-1, n_{2}, w_{1}-l-\Delta, w_{2}-l\right)\right] \\
& +P\left(l, n_{1}, n_{2}, w_{1}-\Delta, w_{2}, 1,0\right)\left(1-(1-\mathrm{BER})^{L_{1}}\right) \\
& \times\left[C_{\text {noise }}^{(1)}+X_{\text {event }}^{(i)}\left(t-l+1-c_{1}-\Delta, n_{1}-1, n_{2}, w_{1}-l-\Delta, w_{2}-l\right)\right] \\
& +P\left(l, n_{1}, n_{2}, w_{1}-\Delta, w_{2}, 0,1\right)(1-\mathrm{BER})^{L_{2}} \\
& \times\left[C_{\text {success }}^{(2)}+X_{\text {event }}^{(i)}\left(t-l+1-s_{2}-\Delta, n_{1}, n_{2}-1, w_{1}-l-\Delta, w_{2}-l\right)\right] \\
& +P\left(l, n_{1}, n_{2}, w_{1}-\Delta, w_{2}, 0,1\right)\left(1-(1-\mathrm{BER})^{L_{2}}\right) \\
& \times\left[C_{\text {noise }}^{(2)}+X_{\text {event }}^{(i)}\left(t-l+1-c_{2}-\Delta, n_{1}, n_{2}-1, w_{1}-l-\Delta, w_{2}-l\right)\right] \\
& +\sum_{k_{1}=2}^{n_{1}}\left[P\left(l, n_{1}, n_{2}, w_{1}-\Delta, w_{2}, k_{1}, 0\right)\right. \\
& \times\left[C_{\text {collision }}^{(1)}+X_{\text {event }}^{(i)}\left(t-l+1-c_{1}-\Delta, n_{1}-k_{1},\right.\right. \\
& \left.\left.\left.n_{2}, w_{1}-l-\Delta, w_{2}-l\right)\right]\right] \\
& +\sum_{k_{2}=2}^{n_{2}}\left[P\left(l, n_{1}, n_{2}, w_{1}-\Delta, w_{2}, 0, k_{2}\right)\right. \\
& \times\left[C_{\text {collision }}^{(2)}+X_{\text {event }}^{(i)}\left(t-l+1-c_{2}-\Delta, n_{1},\right.\right. \\
& \left.\left.\left.n_{2}-k_{2}, w_{1}-l-\Delta, w_{2}-l\right)\right]\right] \\
& +\sum_{k_{1}=1}^{n_{1}} \sum_{k_{2}=1}^{n_{2}}\left[P\left(l, n_{1}, n_{2}, w_{1}-\Delta, w_{2}, k_{1}, k_{2}\right)\right. \\
& \times\left[C_{\text {collision }}^{(1),(2)}+X_{\text {event }}^{(i)}\left(t-l+1-c_{\max }-\Delta, n_{1}-k_{1},\right.\right. \\
& \left.\left.\left.\left.n_{2}-k_{2}, w_{1}-l-\Delta, w_{2}-l\right)\right]\right]\right\}
\end{aligned}
$$

\section{ACKNOWLEDGMENT}

The authors thank the anonymous reviewers who greatly helped to increase the quality of this paper.

\section{REFERENCES}

[1] H. T. Cheng, H. Shan, and W. Zhuang, "Infotainment and road safety service support in vehicular networking: From a communication perspective," Mech. Syst. Signal Process., Special Issue on Integrated Vehicle Dynamics, vol. 25, no. 6, pp. 2020-2038, Aug. 2011.

[2] Amendment 6: Wireless Access in Vehicular Environments, IEEE Std. 802.11p, Jul. 2010.
[3] IEEE Standard for Wireless Access in Vehicular Environments (WAVE) Multi-channel Operation, IEEE Std. 1609.4, Feb. 2011.

[4] N. Wisitpongphan, O. K. Tonguz, J. S. Parikh, P. Mudalige, F. Bai, and V. Sadekar, "Broadcast storm mitigation techniques in vehicular ad hoc networks," IEEE Wireless Commun. Mag., vol. 14, no. 6, pp. 84-94, Dec. 2007.

[5] K. D. Huang, K. R. Duffy, and D. Malone, "On the validity of IEEE 802.11 MAC modeling hypotheses," IEEE/ACM Trans. Netw., vol. 18, no. 6, pp. $1935-1948$, Dec. 2010

[6] C. Campolo, A. Vinel, A. Molinaro, and Y. Koucheryavy, "Modeling broadcasting in IEEE $802.11 \mathrm{p} /$ WAVE vehicular ad hoc networks," IEEE Commun. Lett., vol. 15, no. 2, pp. 199-201, Feb. 2011.

[7] J. B. Kenney, "Standards and regulations," in VANET, Vehicular Applications and InterNetworking Technologies, H. Harteinstein and K. P. Labertaux, Eds. Hoboken, NJ: Wiley, 2010, ch. 9. 
[8] M. Torrent-Moreno, J. Mittag, P. Santi, and H. Hartenstein, "Vehicleto-vehicle communication: Fair transmit power control for safety-critical information," IEEE Trans. Veh. Technol., vol. 58, no. 7, pp. 3684-3703, Sep. 2009.

[9] O. K. Tonguz, N. Wisitpongphan, F. Bai, P. Mudalige, and V. Sadekar, "Broadcasting in VANETs," in Proc. IEEE INFOCOM MOVE Workshop, 2008, pp. 7-12.

[10] R. Schmidt, T. Leinmuller, E. Schoch, F. Kargl, and G. Schafer, "Exploration of adaptive beaconing for efficient intervehicle safety communication," IEEE Netw., vol. 24, no. 1, pp. 14-19, Jan./Feb. 2010.

[11] C. Sommer, O. K. Tonguz, and F. Dressler, "Traffic information systems: Efficient message dissemination via adaptive beaconing," IEEE Commun. Mag., Autom. Netw. Appl. Ser., vol. 49, no. 5, pp. 173-179, May 2011.

[12] DSRC Implementation Guide. A Guide to Users of SAE J2735 Message Sets Over DSRC. [Online]. Available: http://www.sae.org/standardsdev/ dsrc/DSRCImplementationGuide.pdf

[13] Intelligent Transport Systems (ITS); Vehicular Communications; Basic Set of Applications; Part 2: Specification of Cooperative Awareness Basic Service, ETSI TS 102 637-2 V1.1.1, Apr. 2010

[14] X. Ma and X. Chen, "Delay and broadcast reception rates of highway safety applications in vehicular ad hoc networks," in Proc. INFOCOM Workshop, 2007, pp. 85-90.

[15] J. He, Z. Tang, T. O'Farrell, and T. M. Chen, "Performance analysis of DSRC priority mechanism for road safety applications in vehicular networks," Wirel. Commun. Mobile Comput., vol. 11, no. 7, pp. 980-990, Jul. 2011.

[16] A. Vinel, Y. Koucheryavy, S. Andreev, and D. Staehle, "Estimation of a successful beacon reception probability in vehicular ad hoc networks," in Proc. IWCMC, 2009, pp. 416-420.

[17] E. M. van Eenennaam, W. Klein Wolterink, G. Karagiannis, and G. J. Heijenk, "Exploring the solution space of beaconing in VANETs," in Proc. IEEE VNC, 2009, pp. 1-8.

[18] M. I. Hassan, H. L. Vu, and T. Sakurai, "Performance analysis of the IEEE 802.11 MAC protocol for DSRC with and without retransmissions," in IEEE WOWMOM, 2010, pp. 1-8.

[19] J. R. Gallardo, D. Makrakis, and H. T. Mouftah, "Mathematical analysis of EDCA's performance on the control channel of an IEEE 802.11 WAVE vehicular network," EURASIP J. Wireless Commun. Netw., vol. 2010, p. $489527,2010$.

[20] S. Eichler, "Performance evaluation of the IEEE 802.11p WAVE communication standard," in Proc. IEEE Veh. Technol. Conf., 2007, pp. 2199-2203.

[21] G. Badawy, J. Misic, T. Todd, and D. Zhao, "Performance modeling of safety message delivery in vehicular ad hoc networks," in Proc. IEEE WiMob, 2010, pp. 188-195.

[22] E. Schoch and F. Kargl, "On the efficiency of secure beaconing in VANETs," in Proc. IEEE WiSec, 2010, pp. 111-116.

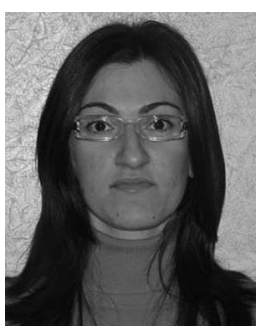

Claudia Campolo (S'10) received the M.S. degree in telecommunications engineering and the Ph.D. degree from the University Mediterranea of Reggio Calabria, Reggio Calabria, Italy, in October 2007 and February 2011, respectively.

In 2008, she was a visiting Ph.D. student with the Department of Electronics Engineering, Politecnico di Torino, Torino, Italy. She is currently a Postdoc Researcher with the University Mediterranea of Reggio Calabria. Her major research interests are in the field of ad hoc and vehicular networking and cooperation in heterogeneous wireless networks.

Ms. Campolo was a corecipient of two best paper awards in the field of vehicular networking.

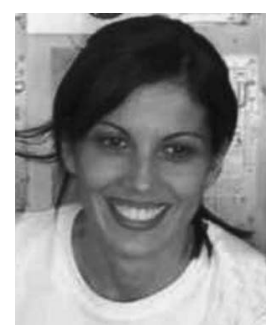

Antonella Molinaro (M'95) received the Laurea (M.S.) degree in computer engineering and the Ph.D. degree in multimedia technologies and communications systems from the University of Calabria, Cosenza, Italy, in 1991 and 1996, respectively, and the Post-Laurea degree in information technology from CEFRIEL/Polytechnic of Milano, Milan, Italy, in 1992.

She joined Telesoft S.p.A., Rome, Italy, as a Telecommunication Network Designer from 1992 to 1993 and the Mobile Network Division Research Center of Siemens, Munich, Germany, as a CEC Fellow for the RACE II mobility action in Advanced-Time Division Multiple Access Mobile Access (ATDMA) project from 1994 to 1995. She was the recipient of a research contract from Polytechnic of Milano and worked in cooperation with Centro Studi e Laboratori Telecomunicazioni, Torino, Italy, in 1997. She was an Assistant Professor of telecommunications with the University of Messina, Messina, Italy, from 1998 to 2001 and the University of Calabria from 2001 to 2004. She is currently an Associate Professor with the University Mediterranea of Reggio Calabria, Reggio Calabria, Italy. Her research interests are mainly in the field of vehicular and ad hoc networks, multihop communications, and future Internet architectures.

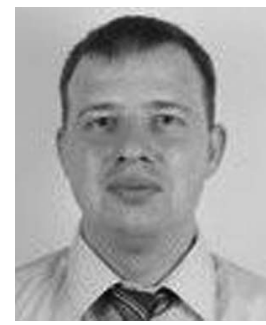

Alexey Vinel (M'07) received the Bachelor (Hons.) and Master (Hons.) degrees in information systems from Saint Petersburg State University of Aerospace Instrumentation, St. Petersburg, Russia, in 2003 and 2005 , respectively, and the Ph.D. degree in technical sciences from the Institute for Information Transmission Problems, Russian Academy of Sciences, Moscow, Russia, in 2007.

$\mathrm{He}$ is currently a Researcher with the Department of Communications Engineering, Tampere University of Technology, Tampere, Finland. His research interests include multiple-access protocols and intelligent transportation systems.

Dr. Vinel has been a Fellow of the Alexander von Humboldt Foundation since 2008 and a member of organizing and technical committees of many international conferences.

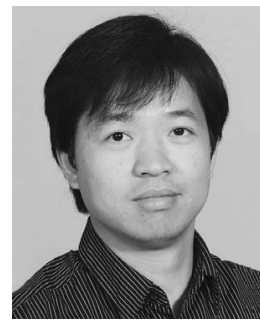

Yan Zhang (M'05-SM'10) received the Ph.D. degree from Nanyang Technological University, Singapore.

Since August 2006, he has been with Simula Research Laboratory, Lysaker, Norway, where he is currently a Senior Research Scientist. He is also an Associate Professor (part-time) with the University of Oslo, Oslo, Norway. His research interests include resource, mobility, spectrum, energy, and data management in wireless communications and networking.

Dr. Zhang is a Regional Editor, Associate Editor, on the Editorial Board, or Guest Editor of a number of international journals. He is currently serving the Book Series Editor for the book series on "Wireless Networks and Mobile Communications" (Auerbach, CRC, Taylor \& Francis Group). He has served as organizing committee chair for many international conferences. 